\title{
A Rare Benign Tumor With Diagnostic Difficulties: Synovial Chondromatosis
}

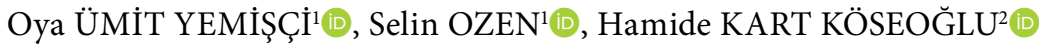 \\ ${ }^{1}$ Department of Physical Medicine and Rehabilitation, Baskent University Faculty of Medicine, Ankara, Turkey \\ ${ }^{2}$ Department of Rheumatology, TOBB ETU University Faculty of Medicine, Ankara, Turkey
}

\begin{abstract}
In this article, we present a case of a 47-year-old male patient presenting with an insidious onset of hip pain and loss of range of motion. The patient was initially treated conservatively to no avail. Detailed investigations included magnetic resonance imaging of the left hip which revealed a synovitis. Blood results were unremarkable apart from moderately raised inflammatory markers. Differential diagnoses of both intra- and extra-articular hip pain were ruled out and the patient treated for a preliminary diagnosis of psoriatic arthritis for a total of six months. Persistent pain resulted in a re-visit of the diagnosis and further clinical evaluation. This time, an X-ray of the hip revealed calcification at the joint. A computed tomography followed and revealed synovial thickening and intra-articular calcification. A biopsy was consistent with primary synovial chondromatosis (SC). Open synovectomy was performed approximately one year after the onset of symptoms. This case emphasizes the importance of re-visiting initial evaluations and diagnoses when faced with a difficult case of persistent hip pain so to avoid misdiagnosis and unnecessary pharmacological treatment. In view of its rarity and diagnostic challenges, future work on SC should concentrate on gathering data which can be used to produce a diagnostic algorithm.

Keywords: Arthritis, chondromatosis, hip, psoriatic, synovial.
\end{abstract}

Primary synovial chondromatosis (SC) is a rare, benign, proliferative metaplastic disorder of the synovium of unknown etiology. ${ }^{1}$ Primary SC affects the knee joint in 55\% of cases, occurs mostly in middle age and shows a slight male preponderance. ${ }^{2,3}$ The hip is the second most commonly affected anatomical site (20\%). ${ }^{3}$ Typically, the patient presents with hip pain and reduced range of motion (ROM) which is not relieved by conservative treatment. ${ }^{4}$

The histological characteristics of primary SC includes cartilaginous nodules of the synovium which are typically intra-articular, but may also occur extra-articularly at the joint bursa or tendon sheath. ${ }^{5,6}$ The first radiographic findings are mostly of a soft tissue swelling indicative of either a joint effusion or synovial-based mass. ${ }^{3}$ More advanced SC may result in radiographic findings of speckled calcification of the femoral head, neck, and acetabulum. ${ }^{7}$ However, due to the non-specific nature of the signs and symptoms associated with $\mathrm{SC}$, and the possible lack of radiological findings, a delay in diagnosis can occur. This in turn can lead to chronic synovial disease and an advancement of arthritic changes and joint damage..$^{8,9}$ In this article, we present a case of primary SC in which mis- and delayed diagnosis occurred due to the lack of positive investigation findings supportive of the diagnosis.

Received: March 07, 2019 Accepted: June 18, 2019 Published online: November 06, 2019

Correspondence: Selin Ozen, MD. Başkent Üniversitesi Tıp Fakültesi Fiziksel Tıp ve Rehabilitasyon Anabilim Dalı, 06490 Bahçelievler, Ankara, Turkey. Tel: +90 530 - 9786262 e-mail: selinhassan@hotmail.com 


\section{CASE REPORT}

A 47-year-old male patient with a three-month history of left hip and groin pain which had become progressively worse over the past month presented to the Physical and Rehabilitation Medicine outpatient clinic. The patient had recently started pilates lessons; the pain was worse on movement and lying on the ipsilateral side. There was no pain at rest and no morning stiffness. The patient had recently been diagnosed with squamous dermatitis and his symptoms of flakey skin on the head and face had reduced following the commencement of treatment with a topical steroid. There was no other drug history or relevant past medical history. A written informed consent was obtained from the patient.

On physical examination, the patient was afebrile, and his vital signs were within the normal range. There was tenderness on palpation of the groin with pain on active and passive internal rotation of the left hip. ROM was within the normal limits. Both flexion abduction external rotation and flexion adduction and internal rotation tests were positive. Lower back ROM was normal with a negative Mennel test. There was no neurological deficit. There was pitting on three of the finger nails. The patient had a minimally antalgic gait. An anteroposterior radiograph of the pelvis and lateral view of the left hip was unremarkable. The patient was given a course of non-steroidal anti-inflammatory drugs for musculotendinous strain and a home exercise program of hip strengthening isometric exercises with a view to follow-up in one month's time.

On follow-up, the patient's left hip pain had continued to worsen despite treatment and a reduction in $\mathrm{ROM}$ had developed. Blood tests revealed a normal full blood count with a raised C reactive protein of $36.8 \mathrm{mg} / \mathrm{L}$. Further rheumatological blood tests were performed and revealed a positive anti-nuclear antibody $(1 / 100$ nucleolar +), a negative rheumatoid factor and anti-cyclic citrullinated peptide antibody. Anti-extractable nuclear antigen antibodies (anti Sjögren syndrome A [Ro], Anti Sjögren syndrome B [La], anti-double stranded deoxyribonucleic acid, anti-Smith, anti-ribonucleoprotein) and human leukocyte antigen B27 surface antigen were also negative.
Magnetic resonance imaging (MRI) of the lumbar spine and sacroiliac joints ruled out the differential diagnoses of lumbar radiculopathy and inflammatory spondyloarthropathy. An ultrasound of the left groin was performed to rule out an inguinal hernia, rectus femoris and adductor muscle rupture. An MRI of the left hip was performed to further rule out differential diagnoses of avascular necrosis, rectus femoris and iliopsoas tendinitis. The MRI revealed an effusion of the left hip, possibly due to synovitis. The patient was advised to use a walking aid. Following discussion with the orthopedics team, ultrasound-guided aspiration of the effusion, using a $18 \mathrm{G}$ needle, under local anesthetic was performed and the resultant $5 \mathrm{~mL}$ of aspirate was sent to the laboratory for culture.

Following a negative culture and gram stain, the rheumatology department was consulted and a course of corticosteroids was started due to the presence of synovitis. Following no resolution of the patient's symptoms, the rheumatology department was re-consulted, the oral corticosteroids were stopped, and methotrexate, salicylazosulfapyridine, diclofenac sodium and a proton pump inhibitor were started as the treatment for a preliminary diagnosis of psoriatic arthritis (PsA).

The medical treatment was continued for a further six months; however, the patient's complaints of left hip pain and reduced ROM worsened; despite an ultrasound-guided intraarticular injection of corticosteroid. A control MRI of the left hip once again revealed an effusion similar to the previous MRI. Three months later, eleven months after initial presentation, a radiograph of the left hip revealed calcification proximal to the head of femur which had not been present previously (Figure 1). A computed tomography revealed synovial thickening, similar to that seen on the previous MRI, and intra-articular calcification (Figure 2). An ultrasound-guided synovial biopsy was taken; macroscopic findings of the biopsy material revealed hyperplastic synovium covering white, multilobulated, nodular projections of hyaline cartilage and microscopic findings included lobules of chondrocytes consistent with a diagnosis of primary SC. No cellular atypia was detected. A year after the initial onset of symptoms, the patient 

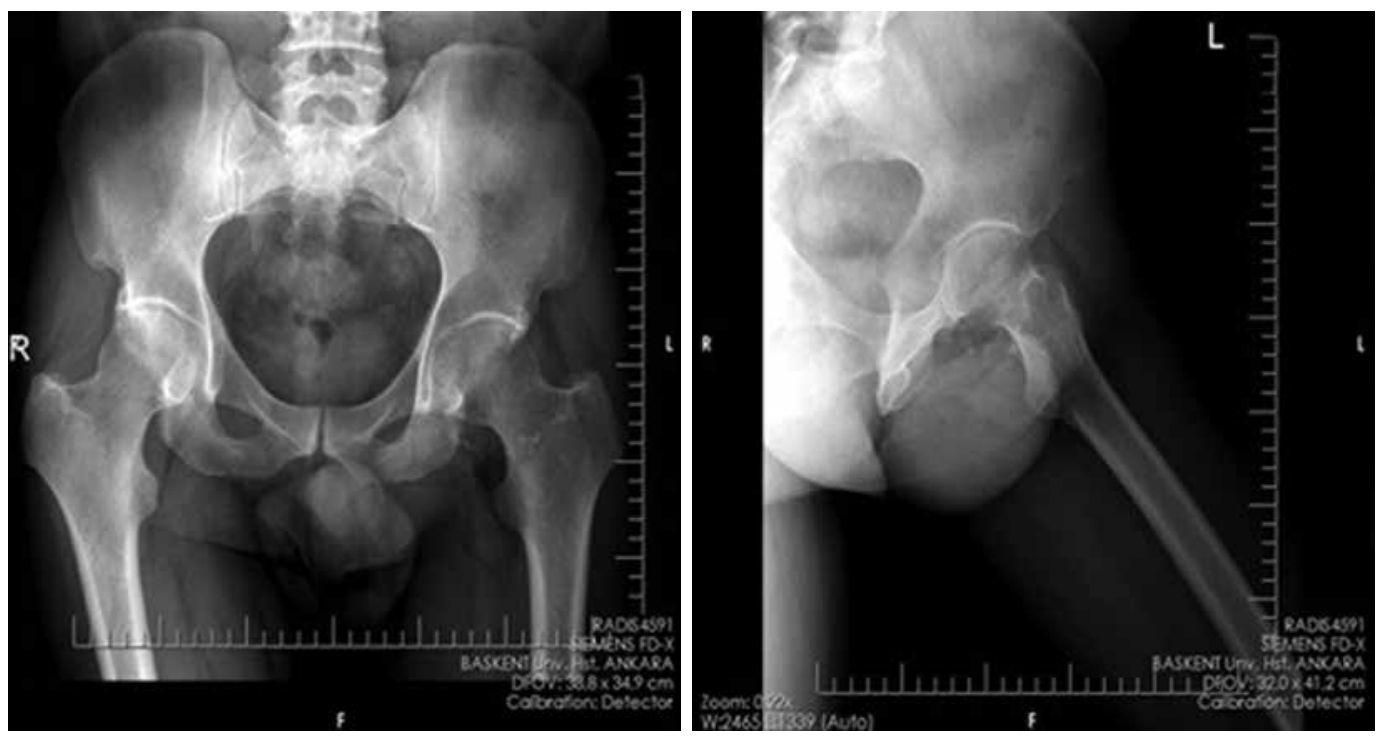

Figure 1. X-ray of pelvis and left hip demonstrating peri-articular calcification.

underwent safe dislocation of the left hip, total synovectomy and removal of the intra-articular osteocartilaginous loose bodies.

The patient was non-weight-bearing for one month postoperatively. An intensive rehabilitation program was commenced postoperatively and is currently continuing. Six weeks postoperatively, the patient remains pain-free and, apart from a minimal decrease in internal rotation, has a normal ROM of the left hip.

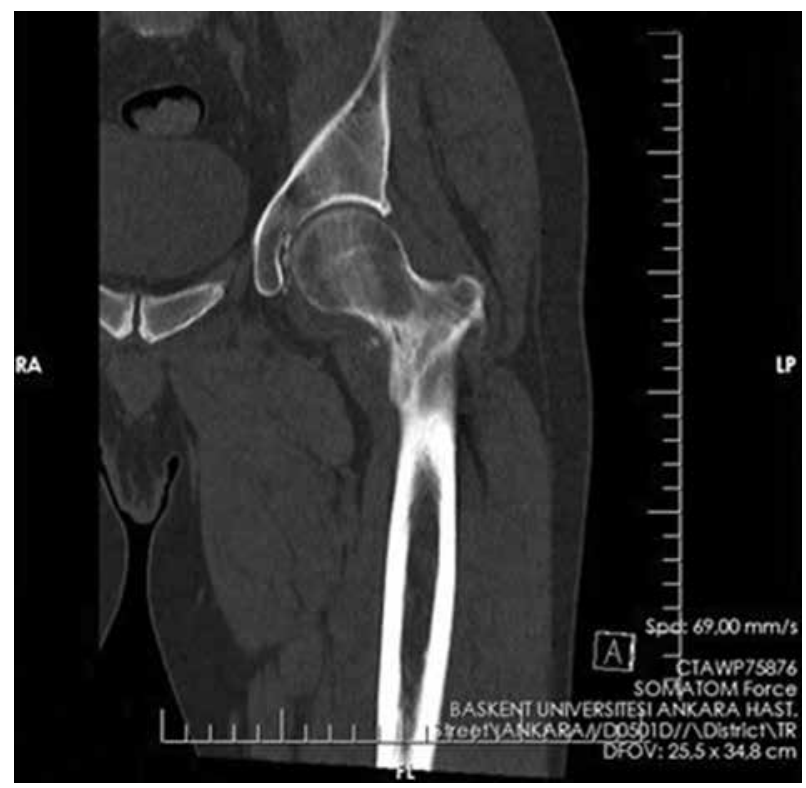

Figure 2. Computed tomography imaging of left hip demonstrating intra-articular calcification.

\section{DISCUSSION}

Primary SC is a rare non-neoplastic proliferative metaplastic disorder of the synovium. ${ }^{1}$ Secondary SC on the other hand is more common, and is associated with mechanical or arthritic joint abnormalities which cause intra-articular chondral bodies. ${ }^{10}$ Due to typical signs and symptoms of SC entailing insidious onset of joint pain (85-100\%), reduced ROM (38-55\%) and swelling (42-58\%), the differential diagnoses include a whole array of intra-articular and extra-articular pathologies such as femoral acetabular impingement, femoral neck valgus/varus deformities, inflammatory joint diseases such as rheumatoid arthritis and spondyloarthropathy. Presence of radiographic evidence of a soft tissue swelling initially may be indicative of either a joint effusion or synovialbased mass which may speed up the diagnostic process. ${ }^{3}$ Radiographic features may also include multiple intra-articular chondral bodies with "ring-and-arc" chondroid mineralization and extrinsic erosion of articular bone. ${ }^{11}$

In this case, there was a delay in diagnosis due to the lack of radiographic findings; there was no soft tissue swelling or calcification suggestive of intra-articular pathology. This further widened the spectrum of differential diagnoses to also include extra-articular pathologies. In turn, ruling out these diagnoses further delayed a positive diagnosis. A systematic literature 
review of SC of the hip by Startzman et al. ${ }^{12}$ reported that radiographic evidence of a soft tissue swelling and calcifications within the joint was present in 55\% of the overall number of cases. However, individual studies reported positive radiographic findings in up to $83 \%$ of cases. ${ }^{7,13,14}$ This suggests that approximately half of SC cases do not initially present with positive radiographic findings and underlines the importance of considering this diagnosis in cases of persistent non-specific hip pain unresponsive to conservative treatment.

The presence of a hip effusion, possibly due to synovitis, on MRI resulted in a misdiagnosis of PsA, on a background of nail pitting and dermatitis. The complexity of PsA, the wide spectrum of clinical disease, and its relapsing and remitting nature result in diagnostic difficulties and also open the way for misdiagnosis, as seen in this case. Indeed, validated diagnostic criteria for PsA do not currently exist. ${ }^{15}$

This case emphasizes the importance of 'going back to basics' and re-visiting our initial evaluation and diagnosis when faced with a difficult case of non-remitting hip pain. Even though repeated imaging is not clinically ideal, in this case, repeating the radiographic imaging did aid in diagnosis. Although primary SC is rare, this case reiterates its importance in the differential diagnosis of persistent hip pain and synovitis and how overlooking such rare diagnoses can result in misdiagnosis and unnecessary pharmacological treatment.

The key to treatment of SC is early and complete debridement. In order to provide an early diagnosis and avoid misdiagnosis, we feel that future work regarding SC should concentrate on gathering data which can be used to produce a diagnostic algorithm.

\section{Declaration of conflicting interests}

The authors declared no conflicts of interest with respect to the authorship and/or publication of this article.

\section{Funding}

The authors received no financial support for the research and/or authorship of this article.

\section{REFERENCES}

1. Murphy FP, Dahlin DC, Sullivan R. Articular synovial chondromatosis. J Bone Joint Surg [Am] 1962;44:77-86.

2. Crotty JM, Monu JU, Pope TL Jr. Synovial osteochondromatosis. Radiol Clin North Am 1996;34:327-42.

3. Wittkop B, Davies AM, Mangham DC. Primary synovial chondromatosis and synovial chondrosarcoma: a pictorial review. Eur Radiol 2002;12:2112-9.

4. Tibor LM, Sekiya JK. Differential diagnosis of pain around the hip joint. Arthroscopy 2008;24:1407-21.

5. Kose MM, Durmus O, Ayhan MY, Batmaz AG. A rare cause of chronic hip pain: Intraarticular synovial chondromatosis. Acta Reumatol Port 2014;39:349-50.

6. Raza A, Kailash K, Malviya A. Rare cause of hip pain in a young girl. BMJ Case Rep 2014;2014.

7. Abolghasemian M, Gharanizadeh K, Kuzyk P, Masdari Z, Fakharian M, Safir O. Hips with synovial chondromatosis may display the features of femoroacetabular impingement. J Bone Joint Surg [Am] 2014;96:e11.

8. Boyer T, Dorfmann H. Arthroscopy in primary synovial chondromatosis of the hip: description and outcome of treatment. J Bone Joint Surg $[\mathrm{Br}]$ 2008;90:314-8

9. Zini R, Longo UG, de Benedetto M, Loppini M, Carraro A, Maffulli N, et al. Arthroscopic management of primary synovial chondromatosis of the hip. Arthroscopy 2013;29:420-6.

10. Villacin AB, Brigham LN, Bullough PG. Primary and secondary synovial chondrometaplasia: histopathologic and clinicoradiologic differences. Hum Pathol 1979;10:439-51.

11. Murphey MD, Vidal JA, Fanburg-Smith JC, Gajewski DA. Imaging of synovial chondromatosis with radiologic-pathologic correlation. Radiographics 2007; $27: 1465-88$.

12. Startzman A, Collins D, Carreira D. A systematic literature review of synovial chondromatosis and pigmented villonodular synovitis of the hip. Phys Sportsmed 2016;44:425-31.

13. Lee JB, Kang C, Lee CH, Kim PS, Hwang DS. Arthroscopic treatment of synovial chondromatosis of the hip. Am J Sports Med 2012;40:1412-8.

14. Lim SJ, Chung HW, Choi YL, Moon YW, Seo JG, Park YS. Operative treatment of primary synovial osteochondromatosis of the hip. J Bone Joint Surg [Am] 2006;88:2456-64.

15. Helliwell PS, Taylor WJ. Classification and diagnostic criteria for psoriatic arthritis. Ann Rheum Dis 2005;64:3-8. 